\title{
6. DYNAMICS OF GASEOUS STREAMS IN CLOSE BINARIES
}

\section{Plavec}

In the early forties, it was found that many spectroscopic peculiarities in close binaries can be explained in terms of gaseous streams within the systems (1, 2, 3, 4,). Joy (5) suggested a simple model of a gaseous ring rotating around the primary component of the Algol-like system RW Tauri. When the high-speed computing machines became available, Kopal (6), Gould $(\mathbf{7}, \mathbf{8})$ and Günther $(\mathbf{9}, \mathbf{r o})$ calculated the trajectories of discrete gas particles ejected from the secondary subgiant components, applying numerical integration of the equations of the restricted problem of three bodies. Prendergast (I I) pointed out that the gaseous streams are relatively dense, the mean free paths short, and mutual collisions frequent, so that the problem requires a hydrodynamical approach. This is undoubtedly true, but a really hydrodynamical treatment requires very large computing machines, and I believe that the discrete-particle approach can still furnish new information, being a first approximation upon which one can develop more rigorous models.

\section{PARTICLE TRAJECTORIES}

An attempt has been made at the Ondřejov Observatory to interpret dynamically and spectroscopically a few selected systems, in particular RW Tauri, U Cephei and U Sagittae. Part of this work has already been published (r2).

For RW Tauri, we adopted a mass ratio of 0.21 and Grant's (r3) absolute dimensions; our unit of velocity is then $223 \mathrm{~km} / \mathrm{sec}$. We computed first trajectories of particles ejected from the first Lagrangian point $(\mathrm{LI})$. Assuming that the Ko IV secondary component fills its critical Roche lobe, it must be expected that the outflow of material is most probable at this point where

$$
\text { RW Tau }-L_{1}-0.2
$$

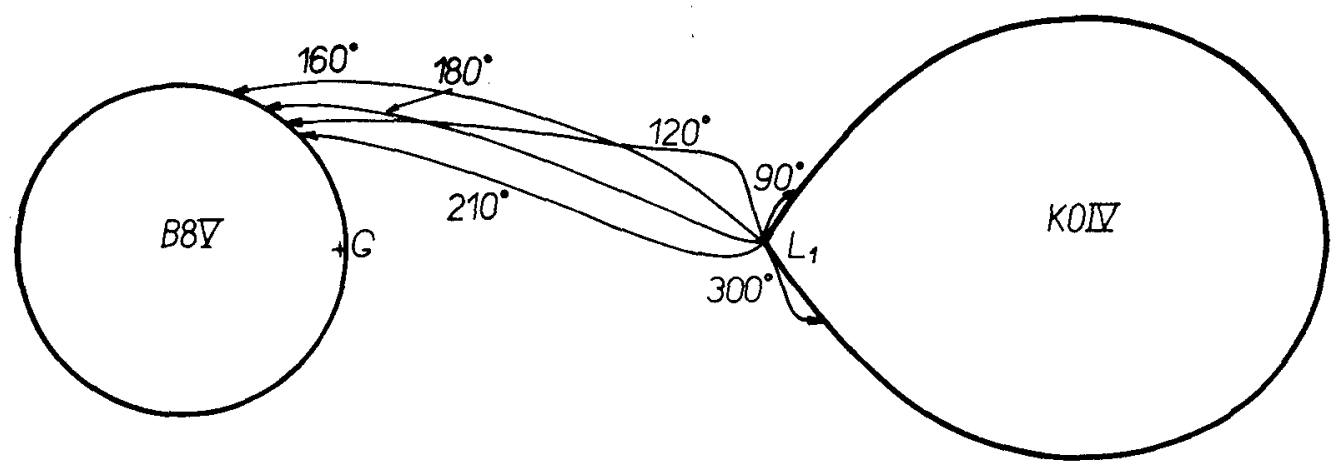

Fig. I. Trajectories of particles escaping from $L_{I}$ with low (thermal) velocities.

the effective gravity vanishes and thermal agitation suffices to expel the particles away from the secondary. However, for velocities of this order, (in fact, up to $0 \cdot 3$ ), all trajectories lead directly to the primary (Fig. I). In the range of ejection velocities from 0.4 to about 0.8 , (i.e. from 85 to $170 \mathrm{~km} / \mathrm{sec}$ ), most of the trajectories also lead directly to the primary, but some are found to 
form one or more rosettes around the primary (Fig. 2 and 3 ). These orbits correspond to ejec tion-velocity vectors lying in the interval of position angles between $125^{\circ}$ and $165^{\circ}$. (Position angles are reckoned counterclockwise, $180^{\circ}$ being directed straightforward to the primary.) Several ring-forming orbits were found also among the three-dimensional orbits, but in general the conditions are more favourable for them in or near the orbital plane.

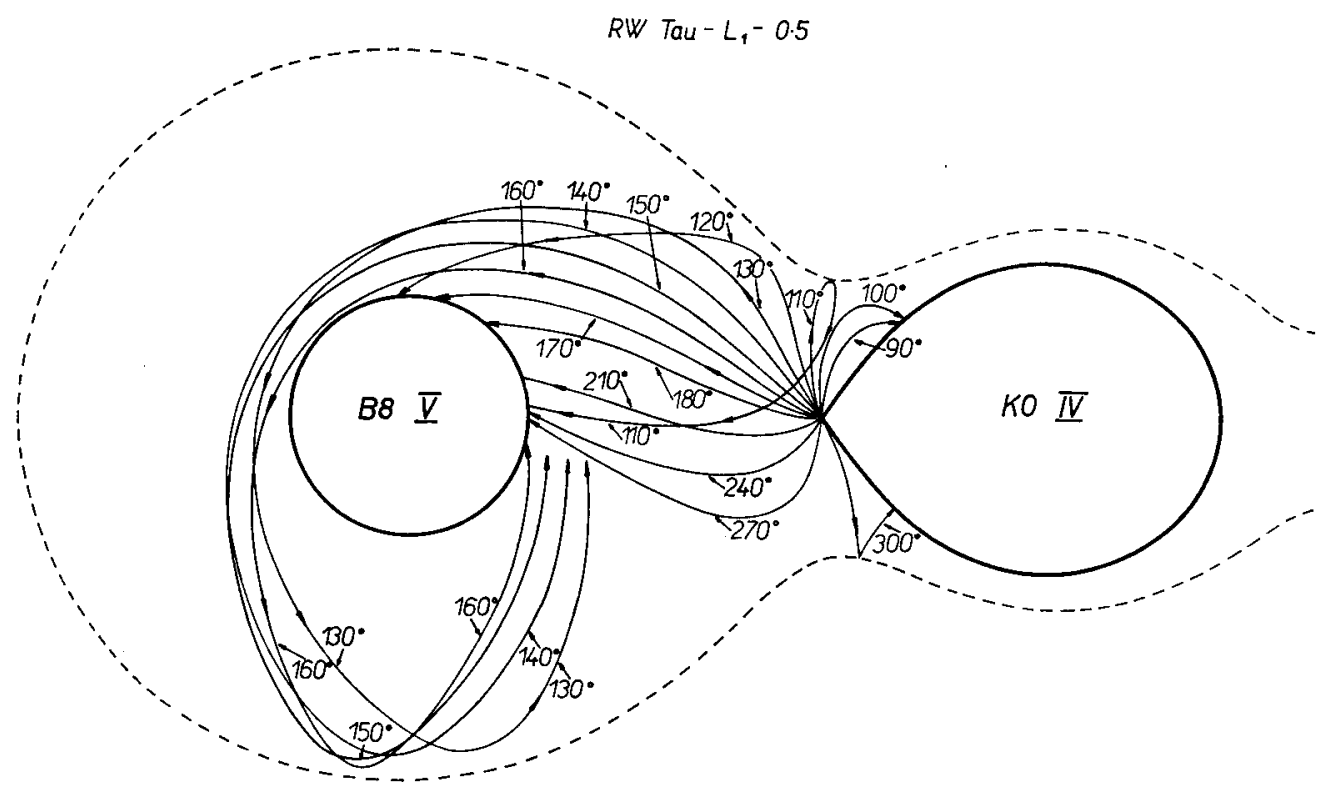

Fig. 2. Ejections from $L_{I}$ with a velocity of $\mathrm{r} r \mathrm{~km} / \mathrm{sec}$.

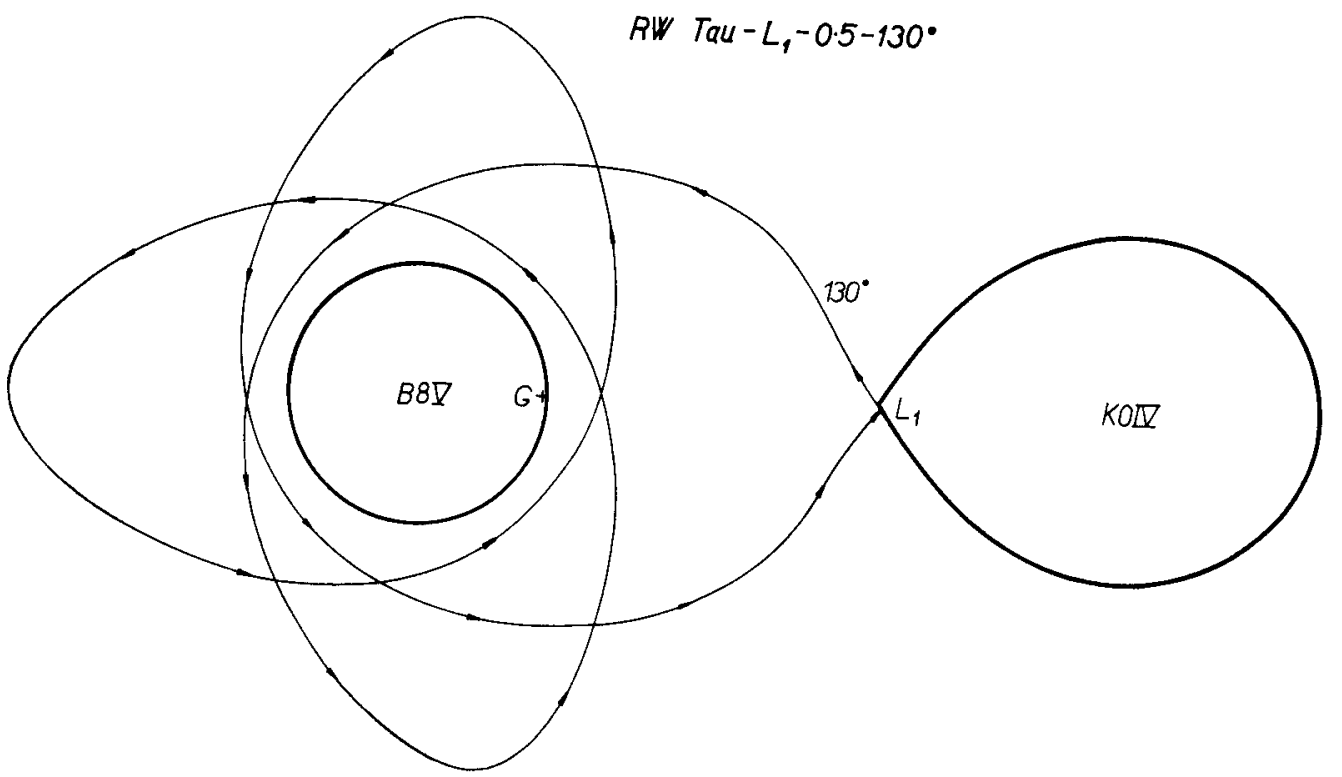

Fig. 3. Example of a ring-forming orbit. 
For ejection velocities higher than $0 \cdot 8$, the character of the orbits gradually changes and there appears a trend towards large retrograde orbits which cannot contribute to the observed rings.

The fact that the ring-forming orbits require relatively high initial velocities implies that we must postulate a certain force operating on the surface of the secondary; if so, the ejections need not be restricted to $\mathrm{L}_{\mathrm{I}}$ only. However, our computations show that only ejections starting in a small region around $L_{I}$ can contribute to the ring around the primary (Fig. 4). Particles

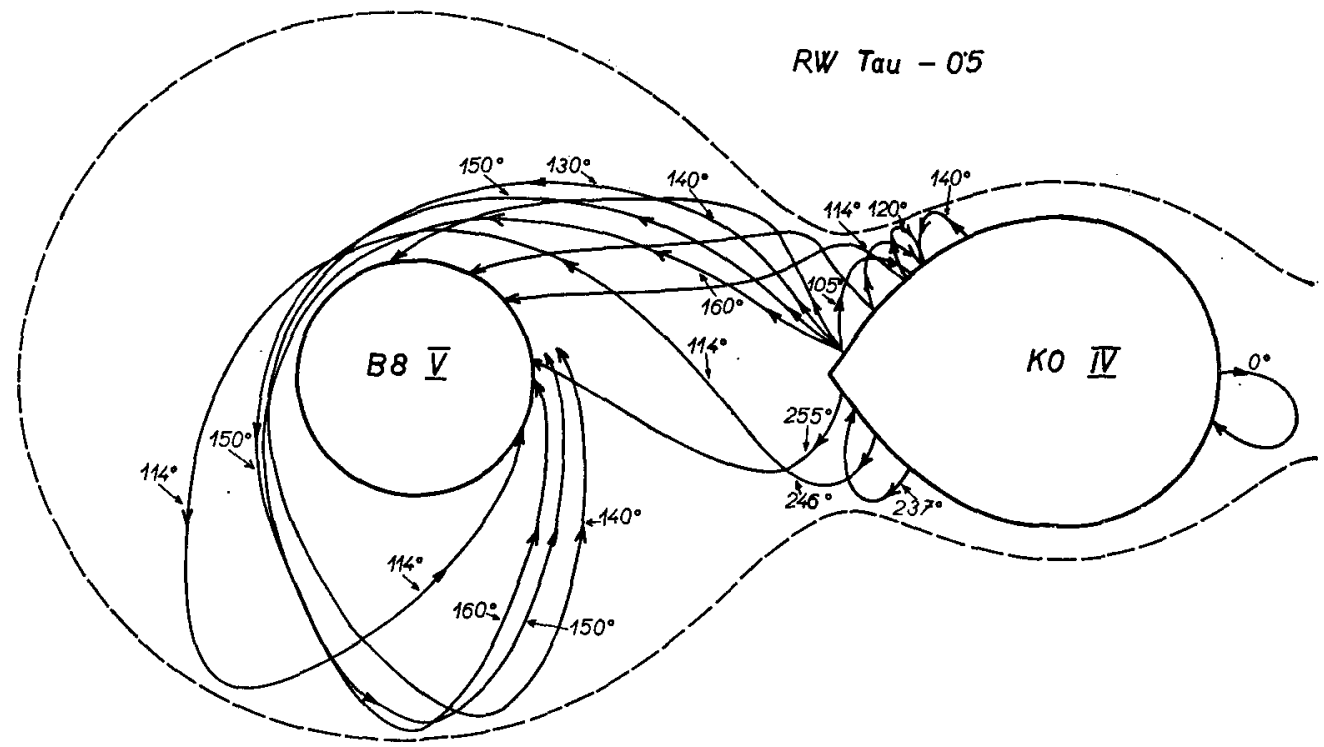

Fig. 4. Ejections at IIo $\mathrm{km} / \mathrm{sec}$ from various parts of the surface of the secondary.

ejected from other parts of the surface of the secondary either fall back or (for higher velocities) fly away along large retrograde orbits.

The picture is very nearly the same also for systems with other mass ratios, as U Sagittae $(0.3)$ and U Cephei $(0.5)$. This result is consistent with observationally established similarity of phenomena in these systems.

\section{COMPARISON WITH OBSERVATIONS: EMISSION LINES}

From the observation of emission lines in RW Tauri, Joy (5, 14) concluded the following: (a) The radius of the ring is not larger than that of the secondary, $(b)$ the intensities of the emission lines vary a great deal, $(c)$ the red-displaced emissions observed near second contact indicate a velocity of $+350 \mathrm{~km} / \mathrm{sec}$, the violet-displaced emissions observed near the end of totality give $-350 \mathrm{~km} / \mathrm{sec}$, $(d)$ from our measurements of Dr Joy's tracings, the width of the red emission lines indicate a velocity dispersion (or turbulence) of about $\pm \mathrm{I} 80 \mathrm{~km} / \mathrm{sec},(e)$ the violet emissions are as a rule weaker and more diffuse.

The character of our computed trajectories accounts well for the properties $(a)$ and $(c)$. It also indicates, in accordance with $(b)$, that the ring is actually a very transient phenomenon, since the lifetimes of particles in the orbits amount to several days only. $(d)$ is probably a consequence of a fairly large extent of the emitting region, over which the orbital velocities vary a great deal.

However, the discrete-particle mechanics fails to account for the violet-displaced emissions. Most of the trajectories are fairly parallel (indicating a laminar flow) over the region observed in 
emission before the totality; but later on, there is a considerable dispersion of individual trajectories leading to many collisions, and the discrete-particle approach fails. The fact that violet-displaced emissions are observed at about the end of totality indicates that the tendency towards a formation of a mean orbit may be quite strong. This could also imply that the ring may be somewhat stabler a phenomenon than our computed lifetimes show. The necessity of a hydrodynamical treatment is quite obvious in this respect.

\section{ABSORPTION EFFECTS}

The gaseous masses encircling the primary produce additional absorption lines when seen projected upon the disk of the primary. The stream lines blend with the normal absorption lines formed in the atmosphere of the primary, making them asymmetrical and causing distortions of the radial-velocity curve (cf., e.g. 15, r6). The hydrogen lines, as well as CaII K, are most affected. It is interesting to see that the asymmetries are prominent at certain phases only, and that there appears to exist a considerable similarity of behaviour among various systems. In U Sge, McNamara (I7) found that there are no asymmetries of the $\mathrm{H}$ lines over the interval of phases $0.36-0.72 p$; after that, strong cores develop displaced towards red from the centre of the wings of the stellar hydrogen lines. Quite similarly, in $\mathrm{U}$ Cep Hardie (16) found no asymmetries between $0.28-0.72$, strong red-displaced cores after 0.75 . In $R$ Arae, Sahade (18) found no asymmetries between $0.45-0.75$, red asymmetries hereafter. Around the phases $0 \cdot 15^{-0.25}$, violet-displaced cores are usually observed, but somewhat less pronounced than the red ones near 0.75 .

An interesting problem is why the effect of the stream is always the strongest near the phases 0.75 and 0.25 . Is this due to a great strength of the stream absorption line at these phases, or due to its large Doppler displacement with respect to the stellar line? This can be only settled by a careful study of line contours, equivalent widths and central depths at various phases. So far the only available observation in this respect is that by Hansen and McNamara (I9) on RZ Scuti; they conclude that the central depths are normal for the spectral type of RZ Scuti A only over the interval of phases $0.26-0.33$. Beginning with 0.33 , the stream absorption develops gradually and attains a great strength after 0.70 .

If we attempt to trace out a mean path of the stream (over the part where the flow is roughly laminar) from our computations, and estimate mean radial velocities of those parts which are projected upon the disk of the primary at various phases, we find that the centre of the stream line should be displaced somewhat to the violet (with respect to the stellar line) at phases preceding 0.62 , and that a strong displacement towards red (amounting to more than roo $\mathrm{km} / \mathrm{sec}$ ) develops after about 0.70 . This is rather an encouraging agreement with the observations; but it is quite evident that a more rigorous hydrodynamical approach will be prerequisite for further progress. Another necessity, however, are high-dispersion spectrograms suitable for microphotometric treatment.

\section{STRUVE'S EFFECT}

What Struve considered to be the most puzzling effect is observed in U Sge and U Cep shortly before the eclipse begins. We know that since about the phase 0.75 , the stream line, appearing as a deep and fairly narrow core superimposed upon the broad stellar hydrogen line, is displaced towards the red with respect to the centre of the wings. But this displacement is seen to diminish after the phase 0.80 , and at about 0.875 the cores reappear on the violet side where they persist until the rotational disturbance sets in. The curious thing is that in spite of this displacement, the radial velocities of the cores still lie above the undistorted radial-velocity curve of the primary. This implies that the broad feature with pronounced wings is actually displaced a great deal above the radial velocity curve, and therefore cannot be identified with 
the normal absorption line formed in the reversing layer of the primary. The phenomenon is observed only in the hydrogen lines (and I suspect also in the $\mathrm{K}$-line of CaII).

Struve $(\mathbf{2 0}, \mathbf{2 I})$ suggested an explanation combining rotation of a tidally distorted star with Stark effect. But McNamara ( $\mathbf{z}$ ) quite justly commented critically on this hypothesis, his main objection being that the primaries are very nearly spherical.

Here an alternative hypothesis will be presented: Calculations of individual trajectories make it probable that a large amount of gas streaming out of the surface of the secondary falls almost directly on the primary, more exactly on those parts of the primary which are observed before the primary eclipse. Near the surface the stream may be dense enough to produce hydrogen lines with appreciable wings. Their radial velocity would be in any case positive, and may easily attain several tens of $\mathrm{km} / \mathrm{sec}$. No similar pheomenon is expected to be present on the opposite hemisphere of the primary, since there is no concentration of orbits there.

It seems that this hypothesis finds observational support in Grant's (13) photoelectric observations of RW Tau. He found an excess light near the beginning of totality which he interpreted as a luminous ring reaching to a distance of about ro per cent of the radius of the primary above its surface, and giving about 0.1 per cent of its total light. He says that a region of higher surface brightness would be an alternative explanation. Nothing of this kind is observed at the end of totality.

Redman (22) observed an unexpected weakening of the hydrogen lines at the limb of the primary components of U Sge and U Cep, and Struve (20) noted changes to occur also in the appearance of other lines at the extreme limb. Perhaps here we could have an insight into the physical state of the suggested 'spurious reversing layer'.

Perhaps another argument in favour of our hypothesis can be found in Struve's observations of $U$ Sge during partial eclipse (20). Between the first and second contact, the hydrogen lines (and also CaII K) are double. The violet component is, according to Struve, probably identical with the violet-displaced stream line observed before the eclipse (from 0.875 ), while the red component, broader and shallower, gives a radial velocity of $+220 \mathrm{~km} / \mathrm{sec}$, much higher than can be expected for any part of the rotating B9 star. Struve says that this line has no counterpart after the end of totality. Struve himself was very near the suggestion that this is also a line originating in a stream, and our opinion is that it is a natural continuation of the broad feature formed in the 'spurious reversing layer'.

This would mean that we actually observe two rather separate gaseous streams. Perhaps the 'directly-falling' stream, responsible for the 'spurious reversing layer' can be identified with the stream observed by Struve and Sahade (23) in emission in the spectrum of Algol at elongations. These emissions are red-displaced and fairly strong near 0.25 , and violet-displaced but quite weak near 0.75 . The displacements are quite understandable if we identify the source with the stream on Fig. 1 . As to the differences in intensity, we can perhaps speculate that near 0.75 we look through the very complex and comparatively dense stream forming the ring (or, better, supplying the ring with fresh material from the secondary), while near 0.25 we may have an essentially unobscured view of the region near $\mathrm{L}_{\mathrm{I}}$.

\section{SOURCE OF GASEOUS STREAMS}

It is probably generally agreed that the gaseous masses observed in Algol-type close binaries come from the secondary component. But what forces are in action there? Kopal (6) suggested that gas particles are ejected at or near Lr provided the rotation of the secondary is not exactly synchronized with its period of revolution. Kopal's idea revived in the work of Kruszewski $(\mathbf{2 4}, \mathbf{2 5}, \mathbf{2 6}$ ), who considers in more detail the model of a secondary which is near its Roche limit and whose rotation is not exactly synchronized. He states mathematically 
the conditions under which a particle travelling up and down the mighty tidal bulges can separate from the star. Kruszewski suggests that in the course of its evolutionary expansion, the secondary attains such dimensions and such a degree of distortion that ejection of material begins at its equator. The initial velocities being small, however, the material falls on the secondary after a short interval of time. Thus there is no actual loss of mass, and the expansion goes on. But this must eventually lead to higher ejection velocities and an actual mass loss. After Kruszewski, the star stops its expansion at a phase when it can transfer mass to the other component, form a gaseous ring around it or produce streams carrying material away from the system.

An advantage of Kruszewski's hypothesis is that this mechanism appears rather natural, since deviations from synchromism are probable, e.g. Struve (27) found that the rotational velocity of $U$ Cep $B$ is $75 \mathrm{~km} / \mathrm{sec}$, whereas the synchronized velocity should be $100 \mathrm{~km} / \mathrm{sec}$. Another advantage is that the positions and initial velocities of the ejected particles are wholly determined by the dimensions and the rotational velocity of the parent star, so that models of gaseous streams can be constructed in more detail.

But a great difficulty is that the deviations from synchronism must be expected to be rather small, which means that the initial velocities will be fairly low. In such cases rings can be formed only around primaries of very small relative radii (under $0 \cdot I$ ). It is doubtful that rings in RW Tau, U Cep or $\mathrm{U}$ Sge could be explained in this way.

For this reason, another hypothesis in terms of rather powerful ejections of material from various regions of the surface of the secondary would seem preferable. We can suggest some analogy to solar activity, perhaps also in the temporal and local distribution of the events.

\section{VARIATIONS OF PERIOD}

Wood (28) pointed out that systems displaying irregular fluctuations of period are all or almost all at the limit of dynamical stability. He suggested that large eruptions eject material at fairly high velocities from various parts of the surface of the unstable components. Then the amount and sign of the change of period depend also on the point of ejection and on the direction of ejection velocity. Wood's hypothesis aroused considerable controversy. It was objected that $(a)$ the mechanism of ejections is doubtful, $(b)$ there is no clear correlation between period fluctuations and character of the system, $(c)$ loss of mass through ejections should lead only to an increase of the period.

However, I think that recent development has been rather decidedly in favour of Wood's hypothesis. First, some kind of ejections is postulated by many other observations. Second, there appears to exist rather a definite connection of period changes with the type of the system. Detached systems with main-sequence components have practically constant periods (apsidal motion disregarded) $(29,30)$, while irregular fluctuations are frequent with semi-detached and constant systems (30). Prichodko (3r) objects to this conclusion and maintains that fluctuations are observed with detached systems too, but her list of such systems contains only Y Cam, RS CVn, Z Her, and AR Lac, which are all systems with subgiants, and further TX Her, whose character is rather doubtful (32).

As to the third of the above-mentioned objections to Wood's hypothesis, several formulae for period variations due to mass transfer were derived in which important terms were neglected, and as a consequence the conclusions were wrong. Formulae derived by Su-Shu Huang (33) show that both increase and decrease of period are possible. A very complete investigation of this problem is due to Piotrowski (34); also Kruszewski (26) derived detailed formulae for circular orbits. Both of them show convincingly that the net change of period depends in about equal extent on the initial conditions, on momentum exchange during flight, and on the $\mathbf{T}$ 
circumstances of landing of the ejected material. Piotrowski finds that exchange of matter introduces a coupling between the orbital and rotational motions of the components: the star losing material tends to restore the synchronism of its rotation, while the star collecting material is generally accelerated in its axial rotation. A small orbital eccentricity generally tends to decrease, while an increase of eccentricity is accompanied by an increase in the separation of the components.

\section{SURVEY OF SYSTEMS WITH GASEOUS RINGS} Sct.

Sahade (35) listed ${ }_{5} 5$ systems with gaseous rings. To his list, we can add U Cep and RZ

It was generally believed that gaseous rings and streams occur in semi-detached systems, i.e. in systems in which the secondary is a subgiant filling its critical Roche lobe. Already Kopal (6) objected to this scheme. Unfortunately, a great deal of the 17 systems have not been observed with an accuracy allowing a definite decision about the character of the system. Nevertheless it seems necessary to change the above statement as follows: Gaseous streams appear to be frequent in systems in which at least one component is above the main sequence. It can be a subgiant filling its Roche lobe (U Cep, U Sge, W Del), an undersize subgiant (RS Cep, VW Cyg, AQ Peg), but also a giant much smaller than the Roche limit in large systems (RX Cas, SX Cas).

In fact, the same conclusion appears to follow from the study of period fluctuations (29): irregular fluctuations or sudden changes occur also in systems where the subgiant is undersized with respect to the Roche limit, so that the system is usually classified as detached.

It is possible to speculate that large eruptions are rather common for stars above the main sequence, but that in the case of close binaries the chances are better to detect the escaping material. The primary would then play a role of a spectroscopic screen. But it must be also affected itself. Sometimes large amounts of material may fall on its surface. We can speculate that there can be a similarity between Algol systems and those dwarf close binaries like U Gem or novae: maybe the different behaviour is due to a different reaction of the primary on the infalling stream of hydrogen gas.

\section{REFERENCES}

r. Kuiper, G. P. Astrophys. F., 93, I33, I94I.

2. Struve, O. Astrophys. $\mathcal{F}$., 103, 77, 1946.

3. Struve, $O$. Observatory, 66, 208, 1946.

4. Struve, O. Stellar Evolution, Princeton Univ. Press, Princeton, $195^{\circ}$.

5. Joy, A. H. Publ. astr. Soc. Pacif., 54, 35, 1942.

6. Kopal, Z. Ann. Astrophys., 19, I, 1956.

7. Gould, N. L. Publ. astr. Soc. Pacif., 69, 541, 1957.

8. Gould, N. L. Astr. F., 64, 136, 1959.

9. Günther, O. Astr. Nachr., 284, 167, 1958.

10. Günther, O. Astr. Nachr., 285, 97, 1959.

II. Prendergast, K. H. Astrophys. F., 132, I62, I960.

12. Plavec, M., Sehnal, L., Mikulaš, J. Bull. astr. Inst. Csl., I5, I71, 1964.

13. Grant, G. Astrophys. F., 129, 62, 1959.

14. Joy, A. H. Publ. astr. Soc. Pacif., 59, I7 I, I947.

15. Struve, O., Huang, S.-S. Handbuch der Physik, Ed. S. Flügge, Springer Verlag, Berlin, 50, $243,1958$.

16. Hardie, R. H. Astrophys. F., I12, 542, 1950.

17. McNamara, D. H. Astrophys. F., 1I4, 5 I3, I95I.

18. Sahade, J. Astrophys. F., 116, 27, 1952. 
19. Hansen, K., McNamara, D. H. Astrophys. F., r30, 791, r959.

20. Struve, O. Mon. Not. R. astr. Soc., 109, 487 , 1949.

21. Struve, O. Popular Astr., 58, 9, 1950.

22. Redman, R. O. Mon. Not. R. astr. Soc., 96, 488, 1936.

23. Sahade, J. Mem. Soc. R. Sci. Liège, 20, 404, $195^{8 .}$

24. Kruszewski, A. Acta astr., 13, 106, 1963.

25. Kruszewski, A. Acta astr., 14, 231, 1964.

26. Kruszewski, A. Acta. astr., 14, 24I, 1964.

27. Struve, O. Publ. astr. Soc. Pacif., 75, 207, 1963.

28. Wood, F. B. Astrophys. F., Ir2, I96, r95०.

29. Plavec, M., Pěkný, Z., Smetanová, M. Bull. astr. Inst. Csl., I1, I81, I960; 12, I 18, I960.

30. Schneller, H. Mitt. Budapest, no. 53, 1962.

31. Prichodko, A. J. Astr. Zu., 38, 927, 196r.

32. Bocula, R. A. Perem. $Z v$., Ir, 26, 1956.

33. Huang, S.-S. Astrophys. $\mathcal{F}$., 138, 471, $48 \mathrm{I}$, 1963.

34. Piotrowski, S. L. Acta. astr., 14, $25 \mathrm{I}, 1964$.

35. Sahade, J. Stellar Atmospheres, Ed. J. L. Greenstein, University Chicago Press, Chicago, $466,1960$.

\section{DISCUSSION}

Piotrowski: I would like to make some comments about gas streams in close binaries when the mechanism of mass ejection results from the non-synchronism of the velocity of rotation of the secondary component with the orbital velocity. A number of trajectories of particles have been computed by Kruszewski in Warsaw and they are shown in the Figures $I$ to 4 for different values of non-synchronism parameter $f(x+f$ is the ratio of the rotational to Keplerian angular velocity), of the parameter $q$ (mass of the companion divided by the mass of the secondary component which is the one losing matter) and for different sizes of the secondary component. One can see from these figures that for every non-stable configuration there exists a gaseous cloud (or stream) asymmetrically placed with respect to the line joining the centres of both components; when the velocity of ejection grows larger, a gas stream develops along which the mass is transferred to the primary and falls on its following hemisphere-like in the gas streams considered by Dr Plavec. It is true-as Dr Plavec noticed it - that the rings forming orbits are only possible when the dimensions of the primary, more massive, component is of the order of one-tenth. But-and this is my further comment-it appears that most often the matter transferred to the primary component tends to increase its rotational momentum. When the amount of transferred matter is large enough, then the equatorial region of the star collecting matter can acquire large rotational velocity subsequently forming a ring which will be enlarged by further transfer of matter.

An additional comment is on the period changes resulting from mass transfer in close binaries. In Table I the values of $\mathrm{d} P / P$ in units of normalized ejected mass are given for several

Table I

\section{Period changes per unit of ejected mass}

$\begin{array}{llll}f & -1.0 & -0.1 & +0.1 \\ q & & & \\ 0.2 & -16.294 & -0.998 & +\mathrm{I} .095 \\ & -12.493 & -7.733 & -6.605 \\ \mathrm{I} \cdot 0 & -5.473 & -0.360 & +0.415 \\ & -0.356 & +0.335 & +0.809 \\ 5.0 & -4.480 & -0.31 \mathrm{I} & +0.369 \\ & +8.915 & +8.69 \mathrm{I} & +9.04 \mathrm{I}\end{array}$




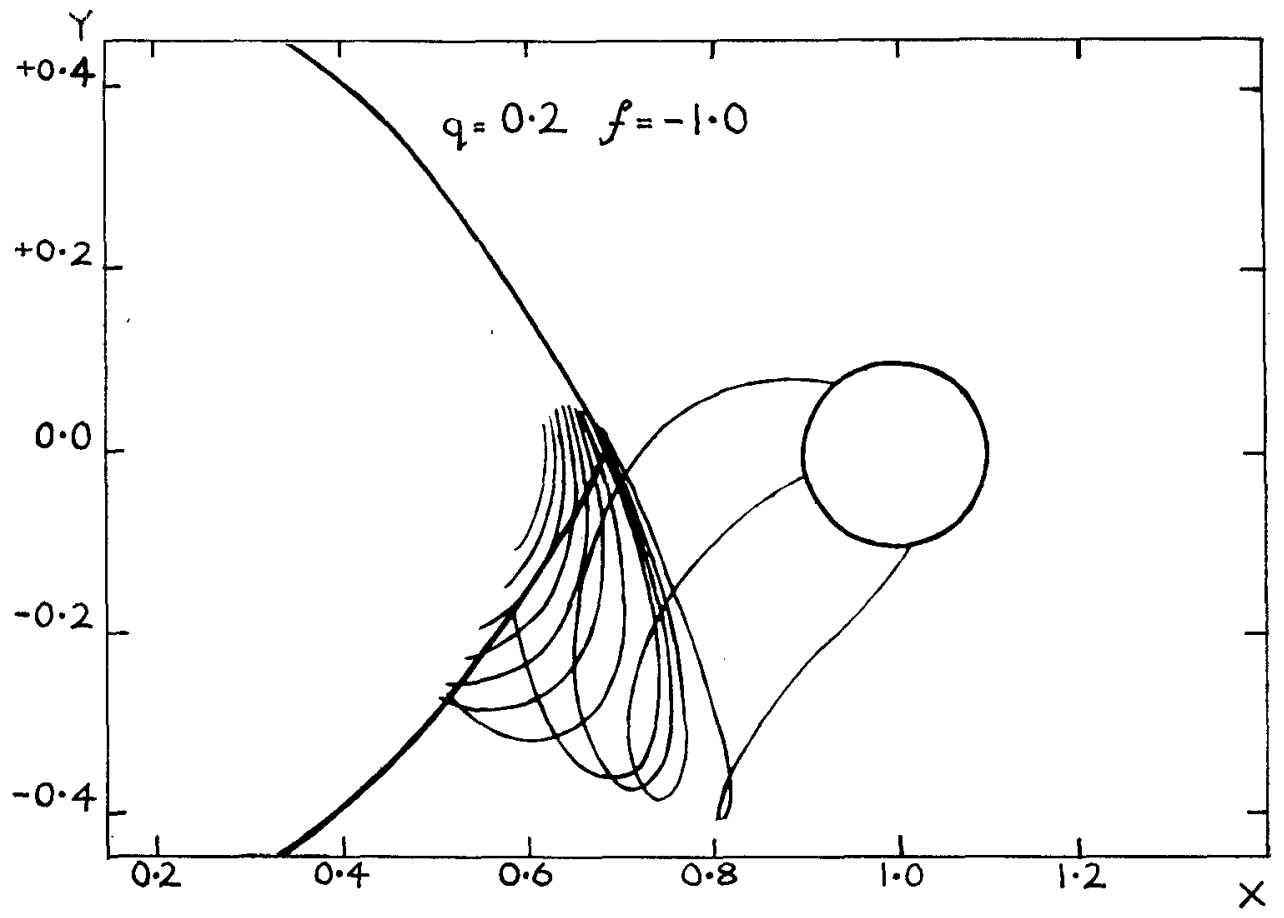

FIG. I

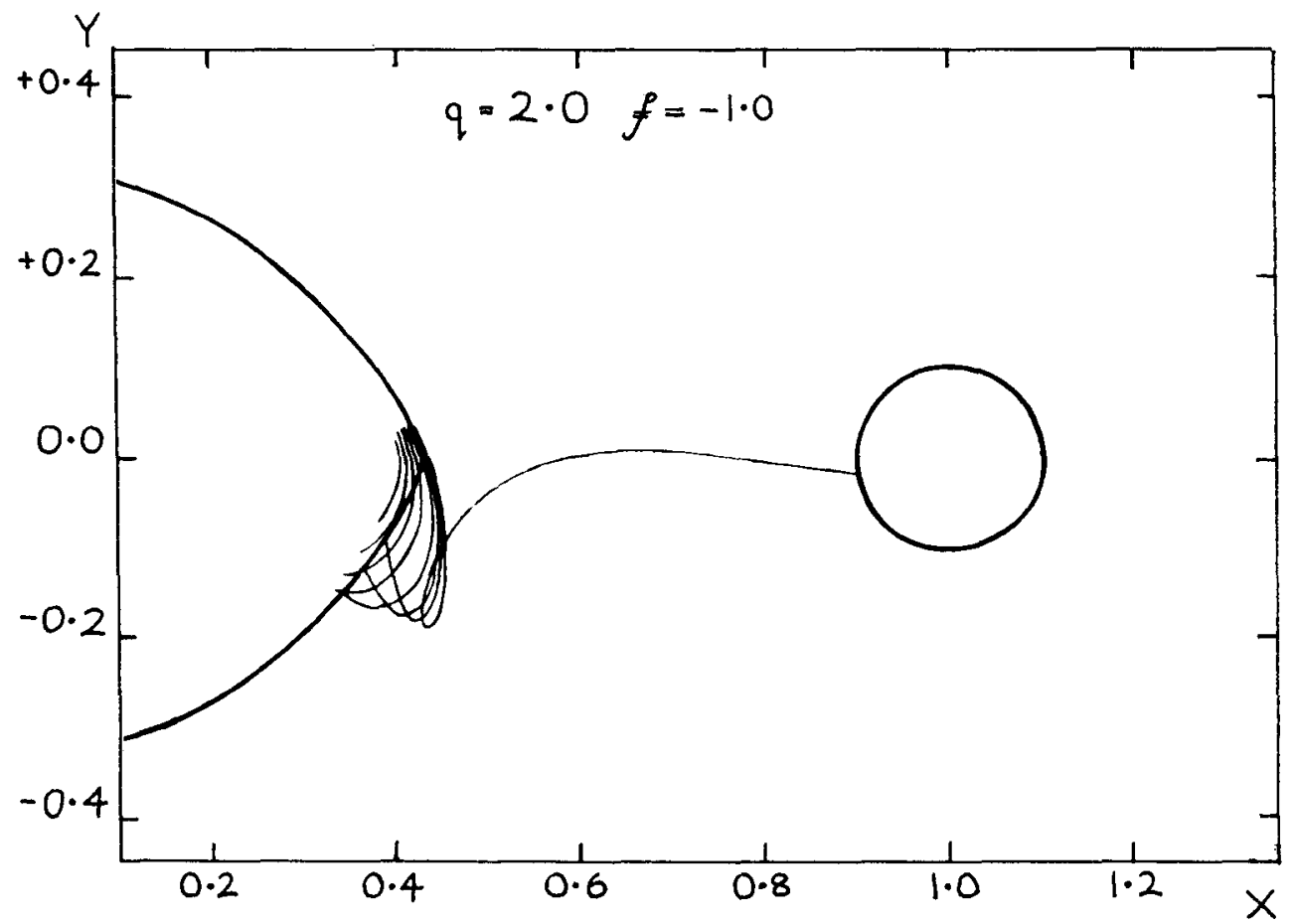

FIG. 2 


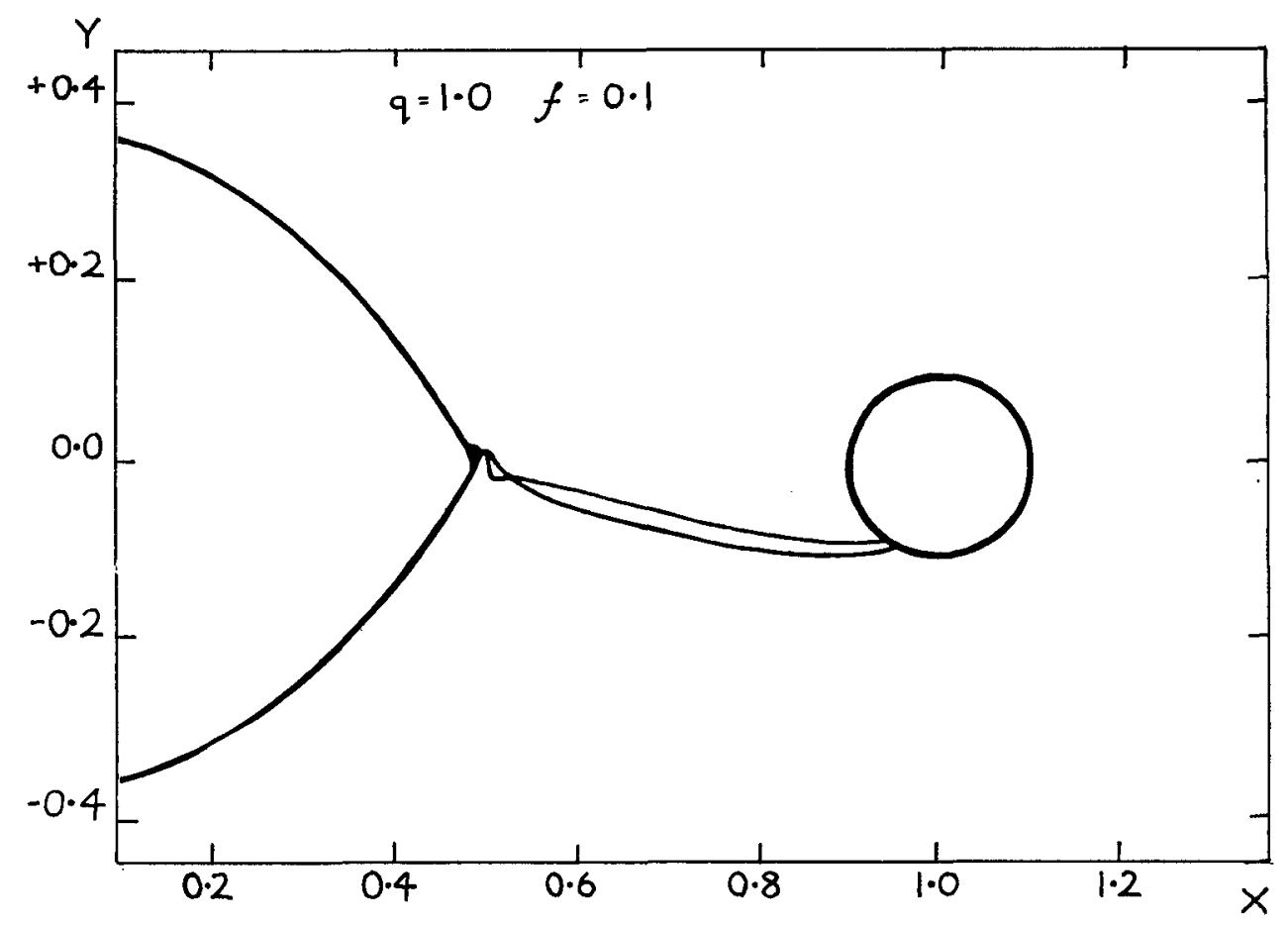

FIG. 3

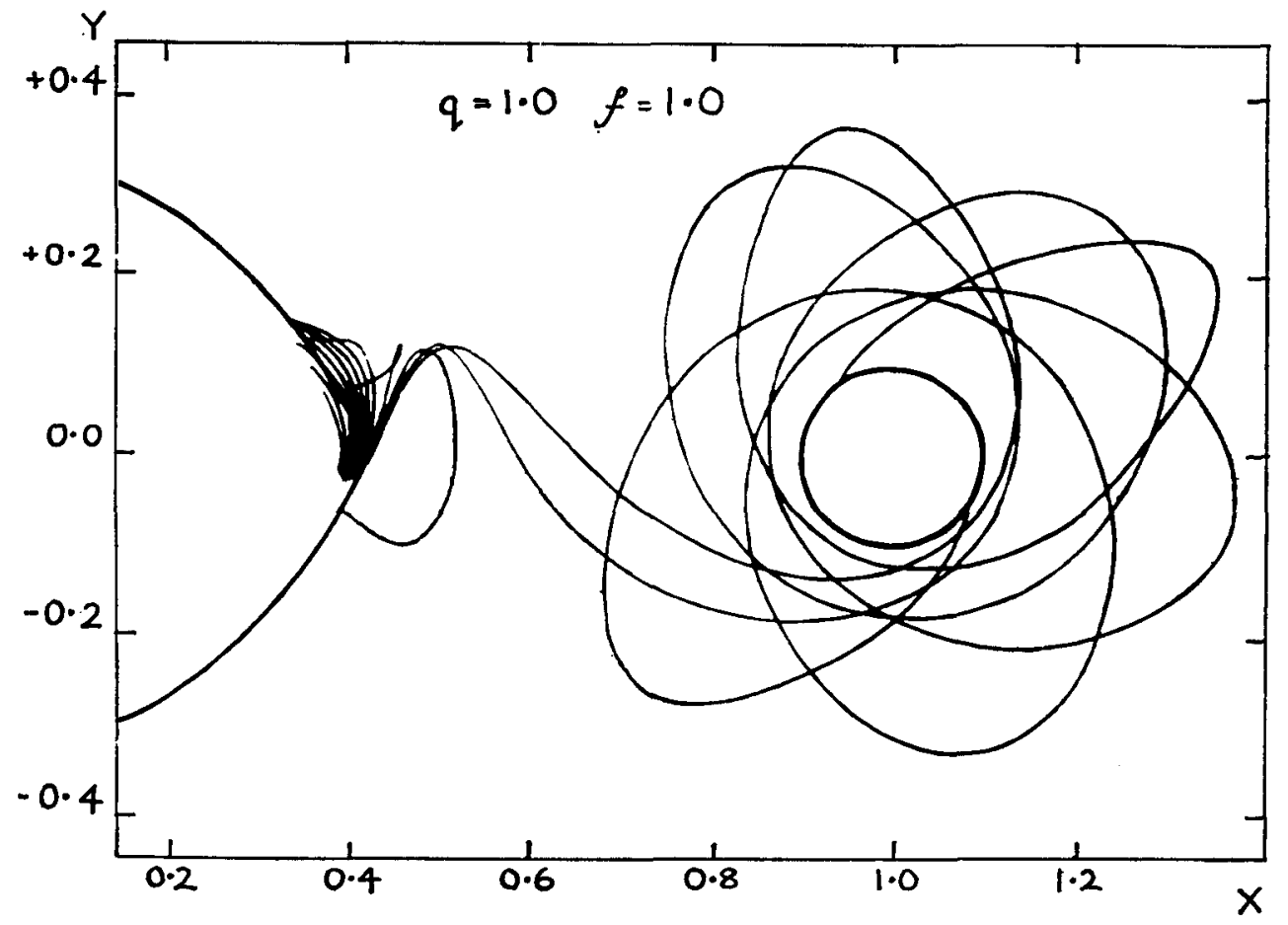

FIG. 4 
values of the parameters $f$ and $q$; for each pair of values $f$ and $q$ two values of $\mathrm{d} P / P$ are given: the upper one corresponding to the case when the element of mass still falls on the same star from which it started, the lower values corresponding to the first trajectory (in the series of trajectories corresponding to increasing velocity of ejection) along which the matter is transferred to the companion. It can be easily seen from this table that for the combination of the parameters $q$ and $f$ most typical for semi-detached eclipsing variables $(q>\mathrm{I}, f<0)$, the period is decreasing as long as there is no transfer of matter to the more massive companion. The resulting period changes depend on the proportion of mass transferred to the mass coming back. If this proportion varies from time to time the period, in principle, can alternately decrease or increase, what is a rather typical behaviour for eclipsing binaries.

My last remark concerns the case of fully detached systems. Also in this case when we have some kind of prominence activity and the ejected matter is falling back onto the same star, we will still have-because of the strong coupling between the rotational and orbital momentaperiod changes; the amount by which the period will change depends of course on the initial and final values of the positions and velocities of the ejected particles as well as on their trajectories.

Gratton: I do not wish to deny the great value of the beautiful computations which were shown. But I think that we should also consider the possible effect of magnetic fields, since even a very weak magnetic field might change the picture completely. Besides, magnetic fields might afford some possible ejection mechanism. After all we know how much the motion of particles ejected by the Sun is affected by the solar, interplanetary and terrestrial magnetic fields: similar effects might well be present in close binaries.

Fracastoro: This is exactly what we are doing at the Observatory of Catania, and perhaps Dr Santarelli will say a few words about the magnetofluidodynamical treatment of the problem.

We also should keep an eye on the Sun, since in several cases the velocity of the particles which travel across its surface is higher than the velocity of escape. This means that magnetic forces are important for our Sun and the same might be true for eclipsing binaries, when trajectories of ejected particles are considered.

Furthermore, in spite that our Sun cannot be considered as a binary star, it shows a differential rotation which can be compared with that of the primary components of eclipsing binaries.

Santarelli: We have investigated, from a magnetofluiododynamic point of view, the instability of a close binary system around the first Lagrangian point. For given values of temperature and density and under the assumptions of non-viscosity and infinite electrical conductivity, we have found a critical value of the magnetic field for the stability, as a consequence of assuming a fixed asynchronism parameter, or vice versa.

Moreover, it can be shown that if the wavelength of symmetrical perturbation exceeds a certain critical value, the contact component can be considered as unstable. The amplitude of this perturbation results, as a rule, greater than twice the major semiaxis of the orbit. Therefore, it seems that the particles ejected by the secondary component may envelop even the whole system before being trapped by the primary component or returning to the secondary component.

Unsöld: Since this is a joint discussion I would like to emphasize the similarity of late-type stars - single and in binaries-with our Sun: (a) Their outermost atmospheres may have temperatures of about a million degrees; that might be important for the transfer of matter in binaries; (b) Late type stars probably have 'plages faculaires' so their $\mathrm{H}_{2}$ and $\mathrm{K}_{2}$ emission lines should exhibit periodic variations due to (i) the rotation of the star and (ii) a 'stellar cycle' analogous to the I I-year solar cycle. 